\title{
Producción de bioetanol carburante a partir del residuo mucilaginoso de cacao CCN-51 y su simulación en Aspen Plus ${ }^{\circledR}$.
}

\author{
Jorge Delgado ${ }^{1,2}$, Jaime Soler ${ }^{2}$, José Ángel Peña ${ }^{2}$ \\ ${ }^{1}$ Universidad Cuenca, Departamento de Química Aplicada y Sistemas de Producción (dQUIMPROd) \\ ${ }^{2}$ Grupo de Catálisis, Separaciones Moleculares e Ingeniería de Reactores (CREG) \\ Instituto de Investigación en Ingeniería de Aragón (I3A) \\ Universidad Zaragoza, Mariano Esquillor s/n, 50018, Zaragoza, Spain. \\ Tel.+34-976762707, e-mail: jorge.delgado@ucuenca.edu.ec
}

\section{Resumen}

La biomasa residual no es suficientemente aprovechada energéticamente en Ecuador. Ejemplo de ello es el mucílago de cacao (CCN-51), en la actualidad un residuo sin aprovechamiento rentable. El objetivo de este estudio fue simular la producción de bioetanol carburante a partir de dicho residuo utilizando el programa Aspen Plus ${ }^{\circledR}$.

\section{Introducción}

El interés por los biocombustibles, en especial para el transporte es cada vez mayor, ya que estos pueden sustituir parcial o completamente a los combustibles fósiles. El agotamiento de estos últimos y su alto coste, sumados al impacto ambiental, resultan ser los factores que promueven la investigación en biocombustibles [1].

La biomasa agrícola residual del Ecuador constituye una fuente renovable de energía con un alto potencial de aprovechamiento. De hecho, en la actualidad, ya se está produciendo la gasolina ECOPAÍs: un biocombustible compuesto por $5 \%$ de bioetanol (proveniente de la caña de azúcar) y un 95\% de gasolina base. Alternativamente, también se analizan otros residuos como los de la palma africana, banano, arroz y cacao.

La simulación del proceso se ha llevado a cabo mediante Aspen Plus ${ }^{\circledR}$, posibilitando la obtención de especificaciones de diseño, condiciones de operación y permitiendo llevar a cabo análisis económicos preliminares de producción industrial.

\section{Metodología}

Para efectos de esta investigación, se propuso la capacidad de diseño para una planta piloto que procese 1000 L/día de mucílago de cacao CCN-51 de la Zona 6 en Ecuador.
Se escogió un modelo NRTL como método de predicción termodinámico en fase líquida no ideal al tratarse de un proceso con componentes polares (etanol, agua, glucosa, etc.) [2].

La etapa de fermentación consistió en dos reactores batch conectados en serie. El primero permite un tratamiento térmico del mucílago de cacao y la inversión de sacarosa. En el segundo se lleva a cabo la fermentación.

Con la solución alcohólica obtenida tras la fermentación, se procedió a realizar el primer proceso de separación del etanol que incluye dos destilaciones con reflujo, obteniendo una solución de al menos 50\% en etanol en una primera etapa y una mezcla cercana al azeótropo en la segunda. Se utilizaron modelos basados en Winn-UnderwoodGilliland para realizar una estimación inicial de los parámetros de operación más relevantes y un modelo "plato a plato" para una simulación rigurosa [3].

La ruptura del azeótropo se lleva a cabo mediante destilación extractiva con glicerina, seguida del uso de tamices moleculares usando Aspen Adsorption ${ }^{\circledR}$ para alcanzar la especificación de etanol anhidro.

\section{Resultados}

El residuo de mucílago de cacao CCN-51 ingresa en la corriente $\mathrm{S} 1$ a temperatura y presión ambientales (Figura 1). En el primer reactor (R-101), de tipo Batch, tiene lugar la reacción de inversión de la sacarosa para aumentar el rendimiento del proceso y proporconar un tratamiento térmico al mucílago para evitar su degradación. Esta reacción ocurre a $127^{\circ} \mathrm{C}$ durante 12 min aproximadamente con un grado de conversión del $90 \%$. A continuación, se hace pasar la corriente de mucílago al reactor R-102 junto con las levaduras de tipo Saccharomyces Cerevisiae para empezar la reacción de fermentación alcohólica de la glucosa a $35{ }^{\circ} \mathrm{C}$ y presión atmosférica durante 48 horas de tiempo máximo. Desde este reactor salen las

Revista “Jornada de Jóvenes Investigadores del I3A”, vol. 8 (Actas de la IX Jornada de Jóvenes Investigadores del I3A -11 de diciembre de 2020). ISSN 2341-4790. 
corrientes de ventilación (VENT), y la corriente S4 con el beer o solución alcohólica.

La corriente VENT elimina en continuo el $\mathrm{CO}_{2}$ producto de la fermentación junto con etanol en equilibrio. Se dirige a una columna de absorción para recuperarlo.

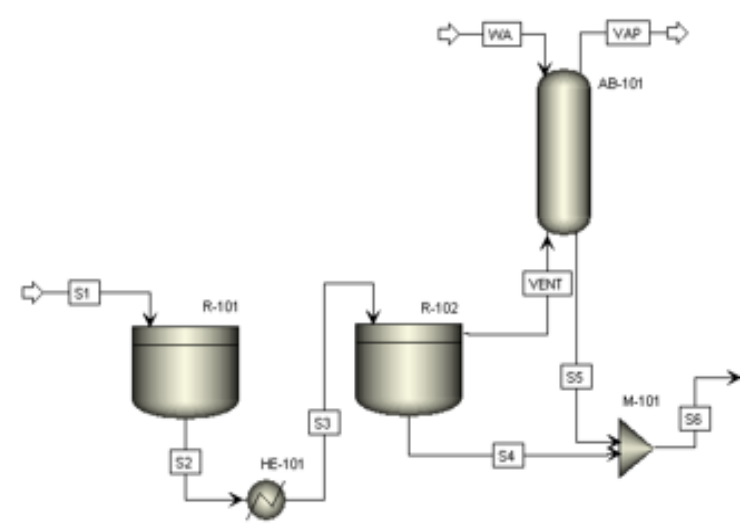

Figura 1.- Sección de reacción del proceso: inversión de la sacarosa, fermentación y absorbedor vapores etílicos.

La Figura 2 muestra el tres de rectificación, que incluye una columna (T-101) con 9 etapas. La corriente de salida del condensador (S10) tiene una concentración másica de etanol del $70 \%$ a $79,67^{\circ} \mathrm{C}$ y 1,02 atm de presión. Dicha corriente ingresará directamente a la etapa 26 de la segunda columna de destilación T-102.

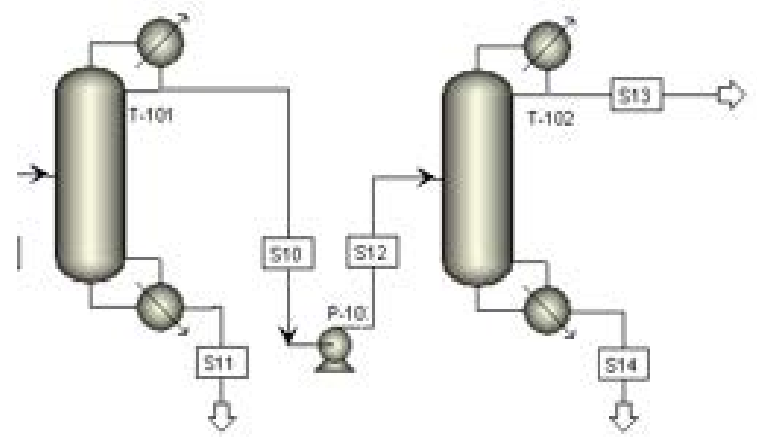

Figura 2.- Detalle del tren de destilación a reflujo para conseguir una mezcla cercana a la azeotrópica.

Posteriormente, la corriente de cabeza de T-102 (S13) se procesa en una columna de destilación extractiva que usa glicerina como solvente extractor (Figura 3). La corriente SOLV lleva aproximadamente $12 \mathrm{~kg} / \mathrm{h}$ de glicerina, que es igual a tres veces la cantidad de alcohol en la corriente de alimentación. La columna de destilación extractiva T-103 cuenta con dos etapas: la primera, que es por donde ingresa el solvente, y la segunda para el etanol concentrado. De aquí sale la corriente S17 que contiene alcohol en un 98\% de fracción másica a 74, $6{ }^{\circ} \mathrm{C}$ y la corriente S16 con la glicerina y el agua separada a $145{ }^{\circ} \mathrm{C}$. Finalmente, la corriente S17 se traslada hasta el lecho de tamices moleculares de zeolita tipo 3A con $0,1 \mathrm{~m}$ de diámetro interno y 0,45 $\mathrm{m}$ de altura por donde es adsorbida la cantidad de agua suficiente para deshidratar el alcohol hasta dejarlo al 99,6\% v/v.

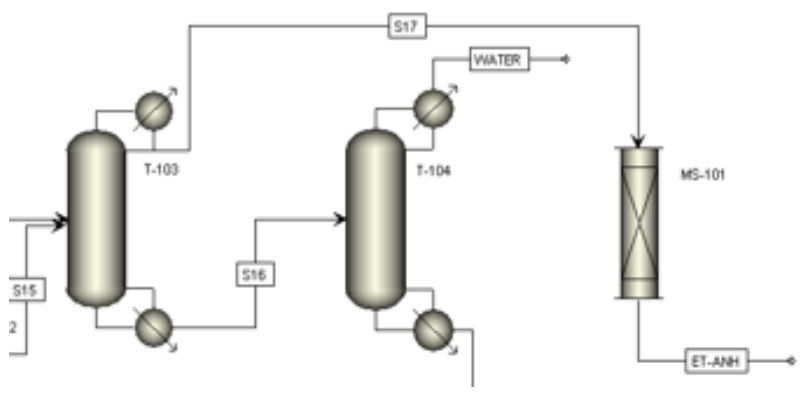

Figura 3.- Tren de destilación extractiva con glicerina y adsorción de zeolita.

\section{Conclusiones}

Se logró simular con satisfacción el proceso de producción de etanol carburante a partir de mucílago de cacao CCN-51 de la Zona 6 de Ecuador como parte de un diseño conceptual.

Se aplicó el software de simulación de procesos Aspen Plus ${ }^{\circledR}$ y se incluyeron el proceso de producción alcohólica (fermentación), destilación con reflujo y deshidratación mediante glicerina.

Además, se empleó Aspen Adsorption ${ }^{\circledR}$ para escalar el proceso de adsorción con tamices moleculares de zeolita tipo 3A para lograr etanol anhidro hasta en un $99,6 \% \mathrm{v} / \mathrm{v}$, porcentaje apto para su uso como aditivo de gasolina en Ecuador.

\section{REFERENCIAS}

[1] Anaya Durand, A., Alarid Miguel, J., Gallegos Diez Barroso, G., \& Sierra Ángeles, J. P. (2006). Heuristics rules for process equipment. Chemical Engineering -New YorkMcgraw Hill Incorporated Then Chemical Week Publishing.

[2] AL-MALAH, K. I. M. (2017). ASPEN PLUS CHEMICAL ENGINEERING APPLICATIONS ( $1^{\text {st }}$ ed.). Wiley. New Jersey.

[3] Cardona, C. A., Sánchez, Ó. J., Montoya, M. I., \& Quintero, J. A. (2005). Simulación de los procesos de obtención de etanol a partir de caña de azúcar y maíz. Scientia et technica, 2(28). https://doi.org/10.22517/23447214.6859

[4] Aspen Technology, Inc. (2018a). Aspen Plus 